\title{
DTL/CDT2 is essential for both CDT1 regulation and the early $\mathrm{G} 2 / \mathrm{M}$ checkpoint
}

\author{
Christopher L. Sansam, Jennifer L. Shepard, Kevin Lai, Alessandra Ianari, Paul S. Danielian, \\ Adam Amsterdam, Nancy Hopkins, and Jacqueline A. Lees ${ }^{1}$ \\ Center for Cancer Research, Massachusetts Institute of Technology, Cambridge, Massachusetts 02139, USA
}

\begin{abstract}
Checkpoint genes maintain genomic stability by arresting cells after DNA damage. Many of these genes also control cell cycle events in unperturbed cells. By conducting a screen for checkpoint genes in zebrafish, we found that $d t I / c d t 2$ is an essential component of the early, radiation-induced G2/M checkpoint. We subsequently found that $d t / / c d t 2$ is required for normal cell cycle control, primarily to prevent rereplication. Both the checkpoint and replication roles are conserved in human DTL. Our data indicate that the rereplication reflects a requirement for DTL in regulating CDT1, a protein required for prereplication complex formation. CDT1 is degraded in S phase to prevent rereplication, and following DNA damage to prevent origin firing. We show that DTL associates with the CUL4-DDB1 E3 ubiquitin ligase and is required for CDT1 down-regulation in unperturbed cells and following DNA damage. The cell cycle defects of Dtl-deficient zebrafish are suppressed by reducing Cdt1 levels. In contrast, the early G2/M checkpoint defect appears to be Cdt1-independent. Thus, DTL promotes genomic stability through two distinct mechanisms. First, it is an essential component of the CUL4-DDB1 complex that controls CDT1 levels, thereby preventing rereplication. Second, it is required for the early G2/M checkpoint.
\end{abstract}

[Keywords: DNA damage; checkpoints; replication; DTL; CDT2; CUL4; CDT1; DCAFs]

Received August 14, 2006; revised version accepted September 25, 2006.

DNA damage is caused by radiation, exogenous genotoxins, free radicals generated during normal cellular metabolism, and errors in cell cycle progression. A critical protective response to DNA damage is to prevent cell cycle progression until DNA is repaired, and failure to properly execute this DNA damage response leads to genomic instability and cancer. In fact, mutations in genes encoding essential components of DNA damage checkpoints cause several inherited cancer susceptibility syndromes such as ataxia telangiectasia (ATM), Seckel syndrome (ATR), Li-Fraumeni syndrome (p53), and Fanconi anemia (FANC proteins) (Kastan and Bartek 2004). To activate cell cycle arrest, complex networks of proteins sense damaged DNA and coordinately inhibit key factors required for DNA replication or the G1/S or G2/M transitions. Importantly, many of the pathways that inhibit cell cycle progression after DNA damage also inhibit cell cycle regulators during the unperturbed cell cycle, thereby maintaining the proper order and timing of cell cycle events.

CDT1 is an example of an essential component of the cell cycle machinery that is inhibited as part of the DNA damage response. CDT1 is required for the formation of the prereplication complexes (pre-RC) that mark poten-

${ }^{1}$ Corresponding author.

E-MAIL jalees@mit.edu; FAX (617) 253-9863.

Article published online ahead of print. Article and publication date are online at http://www.genesdev.org/cgi/doi/10.1101/gad.1482106. tial origins of DNA replication, and proper regulation of CDT1 is essential to ensure that the genome is replicated only once per cell cycle (Bell and Dutta 2002). To prevent rereplication, origins of replication are established in G1, and then pre-RC formation is inhibited during $S$ phase. As an important part of this process, CDT1 is inhibited through two distinct mechanisms. First, a protein called geminin, which appears during $S$ phase and is degraded during mitosis, binds and inhibits CDT1 (McGarry and Kirschner 1998; Wohlschlegel et al. 2000). Second, the ubiquitin protease system (UPS) destroys CDT1 at the onset of S phase (Nishitani et al. 2001; Li and Blow 2004). In addition to the normal cell cycle regulation of CDT1, DNA damage profoundly affects CDT1 protein stability. Exposing cells to ultraviolet or ionizing radiation induces the destruction of CDT1 by the UPS within 15 min (Higa et al. 2003; Hu et al. 2004).

During S phase, two distinct E3-ubiquitin ligases mark CDT1 for destruction by the proteasome (Nishitani et al. 2006; Senga et al. 2006). The first is the SKP1-CUL1-Fbox protein (SCF) complex. SCF is the archetype for the ring-finger/cullin-based class of E3 ligases (Skowyra et al. 1997). It has three constant components-a ring finger protein (ROC1), a scaffold protein (CUL1), and an adapter protein (SKP1). This core complex associates with multiple substrate recognition proteins (F-box proteins) to allow targeting of specific substrates. The substrate rec- 
ognition protein for CDT1 is Skp2 (Liu et al. 2004). The second E3-ubiquitin ligase for CDT1 is the CUL4-DDB1 complex (Arias and Walter 2005). The core structure of this complex is similar to that of SCF; it is comprised of a ring finger protein (ROC1), a scaffold protein (CUL4), and an adapter protein (DDB1). Three different WD40 proteins (AtDET1, CSA, and DDB2) have been identified as specificity factors for the human CUL4-DDB1 complex, but the specificity factor that recruits CDT1 to the CUL4-DDB1 ligase has not been identified (Groisman et al. 2003; Wertz et al. 2004).

In normal cell cycles, the SCF-SKP2 and CUL4-DDB1 complexes have been reported to play overlapping roles in regulating CDT1 stability. Specifically, knocking down CUL4 alone in HeLa cells is insufficient to cause a change in the timing of expression of CDT1, whereas knocking down both CUL4 and SKP2 prevents the degradation of CDT1 during S phase (Nishitani et al. 2006). However, in Caenorhabditis elegans embryos, loss of Cul4 causes rereplication, suggesting that the degree to which SCF-Skp2 or geminin can compensate for Cul4Ddb1 loss is cell-type- or species-specific (Zhong et al. 2003). In contrast to CDT1 regulation during normal cell cycle progression, the destruction of CDT1 after DNA damage specifically requires CUL4-DDB1 (Nishitani et al. 2006). Even in the absence of exogenous DNA-damaging agents, deregulated expression of CDT1 causes profound cell cycle defects. Overexpression of CDT1 or the depletion of geminin causes overt rereplication (as evidenced by $>4 \mathrm{~N}$ cells), DNA damage, and the subsequent activation of a prolonged G2/M checkpoint (Vaziri et al. 2003; Melixetian et al. 2004; Zhu and Dutta 2006).

It is well established that the mammalian G2/M checkpoint is achieved through activation of early and prolonged responses that both impinge upon the mitotic kinase CyclinB/CDC2. The early, transient G2/M checkpoint represents the arrest of cells that were in G2 at the time of damage. Although requirements for this checkpoint are not completely understood, proteins necessary for the initiation (BRCA1 and CtIP) and transduction (ATM and CHK1) of the DNA damage signal are essential for inhibiting mitotic entry within $1 \mathrm{~h}$ of DNA damage (Xu et al. 2002; Zhao et al. 2002; Yu and Chen 2004). The rapid response of this checkpoint results from the inhibition of Cyclin-B/CDC2 through phosphorylation and ubiquitination of upstream regulators including Cyclin B, PLK1, WEE1, CDC25A, CDC25B, and CDC25C (Peng et al. 1997; Sanchez et al. 1997; Raleigh and O'Connell 2000; Smits et al. 2000; Lee et al. 2001; Zhao et al. 2002). In contrast, the late, prolonged G2/M checkpoints take several hours to establish and thus reflect the G2 arrest of cells that were in S phase at the time of damage (Xu et al. 2002; Freie et al. 2004). Two pathways contribute to this late G2/M checkpoint. The first pathway requires p53 and involves the induction of $\mathrm{p} 21^{\mathrm{CIP} 1}$ and the repression of CDC2 and Cyclin B1 (Taylor and Stark 2001). The second pathway requires FANC proteins (FANC-C and FANC-D2) and leads to the downregulation of active $\mathrm{CyclinB} / \mathrm{CDC} 2$ kinase through unknown mechanisms (Freie et al. 2004). Molecular studies have demonstrated that rereplication triggers prolonged G2 arrest through activation of the p53 or FANC pathways (Vaziri et al. 2003; Zhu and Dutta 2006).

Despite the existing complexity of DNA damage networks, it is likely that additional components have yet to be identified. Since zebrafish are amenable to genetic screens, we reasoned that we could use this organism to identify vertebrate DNA damage response genes. We determined that zebrafish embryos respond to IR by activating an ATM/ATR-dependent G2/M checkpoint analogous to the early, transient mammalian G2/M checkpoint, and we screened for zebrafish mutant embryos that failed to activate this checkpoint. This screen led to the identification of two zebrafish lines that have mutations in a gene called denticleless (dt1). We report here that DTL is essential for the early G2/M checkpoint in both zebrafish and human cells. Furthermore, loss of DTL also causes an array of cell cycle phenotypes in unirradiated cells, including rereplication and a delay in G2, which are indicative of a failure to inhibit CDT1 during $\mathrm{S}$ phase. Consistent with these observations, our data show that DTL is a component of the CUL4ADDB1 complex in human cells and is required for CDT1 down-regulation during $S$ phase and following DNA damage. However, our data suggest that deregulation of CDT1 does not itself cause the G2/M checkpoint defect. Therefore, we propose that a CUL4-DDB1-DTL complex also ubiquitinates another key cell cycle regulator in $\mathrm{G} 2$ as part of the activation of the G2/M checkpoint.

\section{Results \\ Ionizing radiation activates a G2 checkpoint in zebrafish embryos}

Vertebrates possess a complex network of proteins and pathways that are required for the DNA damage response (Kastan and Bartek 2004). Many of these factors were identified through genetic screens in lower eukaryotes or as genes disrupted in human developmental and/ or cancer syndromes. We reasoned that we could use the zebrafish to conduct genetic screens for vertebrate DNA damage checkpoint genes. To determine whether we could easily assay a DNA damage checkpoint in zebrafish, we exposed developing embryos to IR and assessed their mitotic index by whole-mount staining with an antibody against phosphorylated (Ser 10) histone H3 (pH3). The phosphorylation of histone H3 predominantly occurs during mitosis, with extensive phosphorylation beginning in late G2, peaking during metaphase and then declining through anaphase (Hendzel et al. 1997). At 32 h post-fertilization (hpf), 3\% of the cells in unirradiated zebrafish embryos were intensely stained with an anti-pH3 antibody (Fig. 1A,F). To visualize mitotic chromatin and spindles, we stained embryos with the anti-pH3 and anti- $\alpha$-tubulin antibodies, respectively. The majority of the $\mathrm{pH} 3$-positive cells had condensed chromatin and mitotic spindles, confirming that these cells were mitotic (data not shown).

We next tested whether exposure to IR would decrease 
A

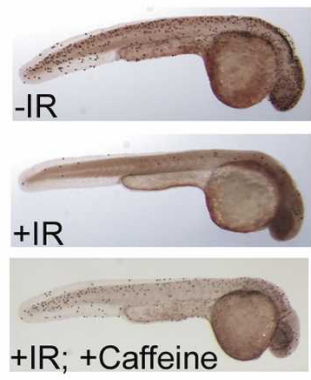

B

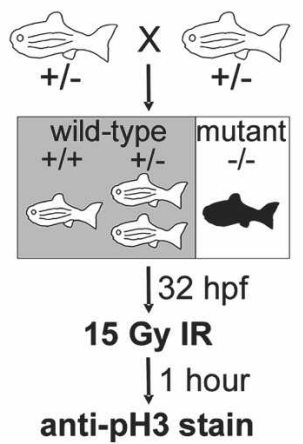

C

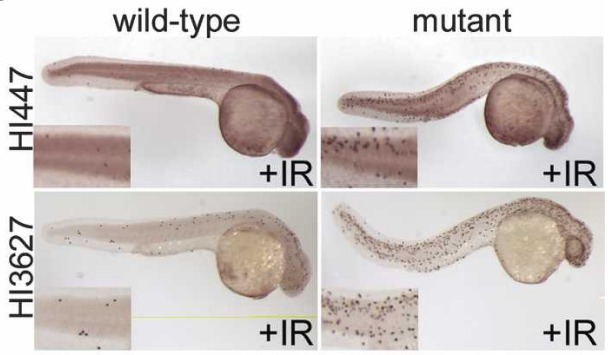

D

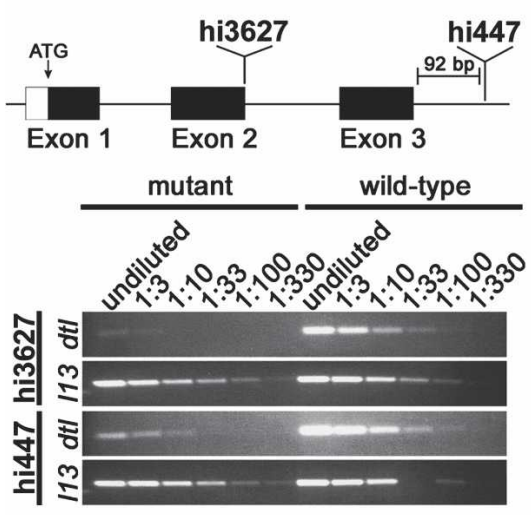

E

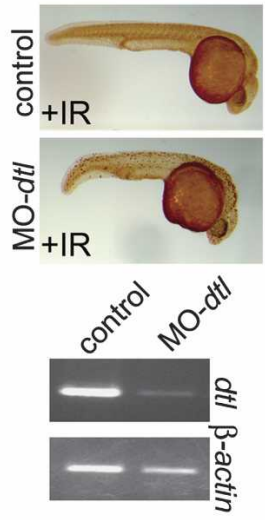

F

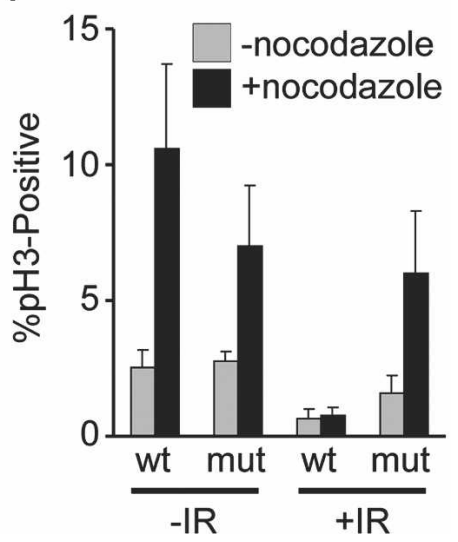

Figure 1. Insertional mutations in the zebrafish $d t 1$ gene cause a G2 DNA damage checkpoint defect. $(A)$ An early G2 checkpoint response was verified by labeling mitotic cells using an anti-pH3 antibody in untreated (-IR), IR-treated (+IR), and caffeine-pretreated/ IR-treated (+IR; +Caffeine) 32-hpf wild-type zebrafish embryos. (B) Scheme of the shelf screen used to identify recessive lethal insertional mutants with G2 checkpoint defects. $(C)$ Analysis of embryos derived from hi3627 and hi447 heterozygous crosses showed a presence of a robust early G2 checkpoint response in wild-type $(+/+;+/-)$ but not mutant $(-/-)$ clutchmates. $(D)$ The positions of the hi447 and hi3627 insertions relative to the first three exons of the zebrafish $d t 1$ gene. Analysis of total mRNA by RT-PCR showed decreased $d t 1$ mRNA, relative to the mrpL13 loading control, in 28-hpf hi3627 and hi447 mutant embryos. $(E)$ dtl morpholinos reduce $d t 1$ mRNA levels and impair the IR-induced G2 arrest in 28-hpf embryos. (F) Nonirradiated (-IR) and irradiated (+IR) wild-type (+/+; $+/-)$ and hi3627 mutant (-/-) clutchmates were maintained in the absence or presence of nocodazole for $2 \mathrm{~h}$, and the percentage of pH3-positive cells was quantified by FACS (mean \pm SD; $n=20,000$ counts in each of three embryos).

the mitotic index. pH3-positive cells almost completely disappeared from irradiated embryos within $1 \mathrm{~h}$ of the IR exposure (Fig. 1A). We have demonstrated that the average duration of G2 in embryos at this developmental stage is longer than $1 \mathrm{~h}$ (see below). Therefore, the loss of $\mathrm{pH} 3$-positive cells $1 \mathrm{~h}$ after ionizing radiation exposure represents a G2 checkpoint. In human cells, a rapidly initiated G2 checkpoint requires the ATM kinase (Xu et al. 2002). To further characterize this DNA damage checkpoint in zebrafish, we tested whether checkpoint activation could be disrupted by treatment with caffeine, an ATM/ATR inhibitor (Sarkaria et al. 1999). While the IR-induced loss of pH3-positive cells was unaffected by treatment with the vehicle alone, the caffeine-treated, irradiated embryos maintained a similar level of $\mathrm{pH} 3-$ positive cells to the unirradiated controls, indicating that the ATM/ATR kinases are required for this checkpoint (Fig. 1A). Thus, zebrafish embryos respond to IR by activating an early, ATM/ATR-dependent G2/M checkpoint similar to their mammalian counterpart. Impor- tantly, these experiments show that a dysfunctional checkpoint is easily scored using the IR-pH3 assay.

\section{A genetic screen for zebrafish mutants with a defective IR response}

We have previously generated a collection of $\sim 500$ zebrafish lines that carry stable viral insertions (Golling et al. 2002). These lines are fully viable as heterozygotes; however, when the viral insert is brought to homozygosity, we have observed developmental defects between 1 and $5 \mathrm{~d}$ post-fertilization that were typically lethal. In most cases, the developmental defect has been linked to a proviral insert within a specific gene. The collection contains mutations within 335 different genes, one-fifth of which have unknown functions (Amsterdam et al. 2004). Many of the known genes have cell-essential functions including regulating cell viability or proliferation, but the homozygous mutants survive for a few days postfertilization because of maternally contributed stores. 
This is notable because several mammalian checkpoint genes, including ATR and Chk1, are also required for cell viability and proliferation (Liu et al. 2000; Takai et al. 2000; Brown and Baltimore 2003).

We conducted a pilot screen on 75 lines from the Hopkins insertional mutant collection. These were selected because they had detectable developmental defects at 36 hpf but remained viable. We also focused on lines that contained mutations within genes of unknown function. For the screen, we collected 50 or more embryos from each heterozygous intercross, and then looked for lines in which approximately one-quarter of the clutch retained a high degree of $\mathrm{pH} 3$ staining after IR treatment (Fig. 1B). We then PCR-genotyped the embryos to determine whether the defective response occurred in embryos homozygous for the mutant insert. Using this screen, we found that hi3627 (Hopkins insertion line 3627) homozygotes failed to show the normal decrease in pH3-positive cells following IR exposure (Fig. 1C). In contrast, the hi3627 heterozygotes respond like their wild-type clutchmates (data not shown). Costaining with anti-pH3 and anti- $\alpha$-tubulin antibodies confirms that the pH3-positive cells in the hi3627 homozygous mutants are mitotic (data not shown). Thus, we refer to this phenotype as Mitosis After Irradiation (MAI).

The hi3627 mutants have general developmental defects, including apoptosis in the central nervous system and a dorsally curved tail, which appear by $28 \mathrm{hpf}$ (Fig. 1C; Golling et al. 2002). Injection of a morpholino that blocks p53 translation is sufficient to delay these developmental phenotypes by $\sim 12 \mathrm{~h}$, indicating that p53 contributes to the ensuing apoptosis (data not shown). By performing the IR-pH3 assay at earlier developmental time points, we found that the MAI phenotype in the hi3627 mutants occurs as early as $16 \mathrm{hpf}$, well before the appearance of any developmental defects (data not shown). Thus, the MAI defect precedes the p53-dependent apoptosis.

The hi3627 line carries a retroviral insertion at the splice donor of exon 2 in the zebrafish denticleless homolog gene $(d t 1)$. The $d t 1$ gene was described in Drosophila melanogaster as a heat-shock-responsive gene that was required for embryonic development, and a highly similar gene exists in Schizosaccharomyces pombe called cdt2 (Cdc10-dependent transcript 2) (Kurzik-Dumke et al. 1996). In the $d t 1^{\text {hi3627 }}$ mutants, the $d t l$ mRNA is barely detectable by RT-PCR at $28 \mathrm{hpf}$ (Fig. 1D). Importantly, the Hopkins mutant collection contains a second $d t l$ mutant line, hi447, in which the retrovirus inserted into the third intron of the $d t l$ gene (Fig. 1D). The developmental defects of the $d t t^{\text {hi3627 }}$ and $d t l^{\text {hi447 }}$ embryos are similar, and RT-PCR analysis confirms that the hi447 viral insert also substantially reduces $d t 1$ mRNA abundance (Fig. 1D). Consistent with the notion that $d t l$ disruption caused the MAI phenotype, the hi447 homozygotes also failed to show the normal decrease in mitotic index after IR exposure (Fig. 1C). As further verification, we knocked down $d t 1$ mRNA using a morpholino oligonucleotide that targets the exon 6 splice donor in the $d t 1$ pre-mRNA. Injection of the $d t 1$ morpholino at the two-cell stage causes a partial reduction in $d t 1$ mRNA levels by $28 \mathrm{hpf}$ and yielded a high mitotic index following IR exposure (Fig. 1E), underscoring our conclusion that the MAI phenotype results from a reduction in $D t l$ expression.

We reasoned that the MAI phenotype in the $d t 1 \mathrm{mu}-$ tant lines could arise through two possible mechanisms: a failure to prevent entry into mitosis following DNA damage or a failure to efficiently exit mitosis. To distinguish between these two possibilities, we performed nocodazole-trapping experiments. Specifically, $d t 1^{\text {hi3627 }}$ mutants and wild-type clutchmates were irradiated and then incubated with or without nocodazole for $2 \mathrm{~h}$ before quantifying the level of pH3-positive cells by FACS (Fig. $1 F)$. Nocodazole treatment causes early to mid-mitotic arrest; hence, an accumulation of $\mathrm{pH} 3$-positive cells during nocodazole treatment demonstrates that cells are entering mitosis. The level of $\mathrm{pH} 3$-positive cells was similar in untreated wild-type versus $d t 7^{\text {hi3627 }}$ mutants, and nocodazole treatment increased the percentage of $\mathrm{pH} 3$ positive cells by fourfold in wild-type embryos and threefold in mutant embryos (Fig. 1F). This confirms that nocodazole treatment inhibits mitotic exit in both wildtype and $d t t^{\text {hi3627 }}$ mutant embryos. As described above, IR treatment greatly reduced the level of pH3-positive cells in the wild-type zebrafish (Fig. 1F). This was completely unaltered when the embryos were placed in nocodazole immediately after irradiation, indicating that the G2 checkpoint is activated rapidly. In contrast, the irradiated $d t l$ mutants retained a high mitotic index without nocodazole, and this was further elevated by nocodazole treatment. These data demonstrate that the MAI phenotype of the $d t l$ mutants reflects a genuine defect in their ability to arrest in G2 following DNA damage.

\section{Disruption of dtl causes multiple cell cycle defects}

DNA damage checkpoint genes are often required for normal cell cycle progression. Consistent with this principle, further analysis of the hi3627 and hi447 lines revealed that the unirradiated $d t l$ mutants had a variety of cell cycle abnormalities. First, FACS analysis revealed that cells derived from 24-hpf $d t t^{\text {hi3627 }}$ and $d t t^{\text {hi4447 }}$ mutant embryos had a dramatic increase in the percentage of cells with $>4 \mathrm{~N}$ DNA content (Fig. 2A). We did not observe a significant number of polyploid cells; instead, the majority of the aneuploid cells had between 4N and $8 \mathrm{~N}$ DNA. This $>4 \mathrm{~N}$ DNA suggests a partial rereplication of the genome. We have also observed this phenotype in $d t 1$ morpholino-injected embryos (morphants), albeit at later time points (36 hpf) and with reduced penetrance consistent with the higher residual levels of $d t 1$ mRNA in morphants versus insertional mutants (Fig. 1, cf. D and E). Partial rereplication is a frequently observed phenotype when pre-RC assembly factors such as CDT1 are not inhibited appropriately during $S$ phase; therefore, these data suggested that Dtl was necessary for inhibiting Cdt1 after DNA replication initiation (Vaziri et al. 2003; Melixetian et al. 2004; Zhu et al. 2004). 
A

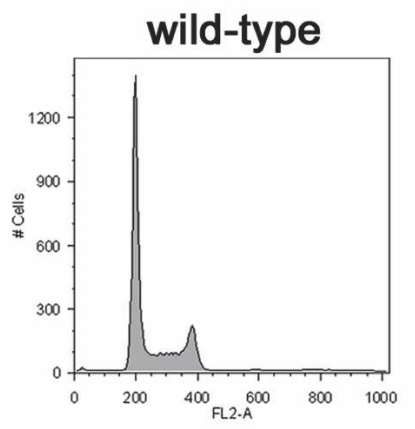

C

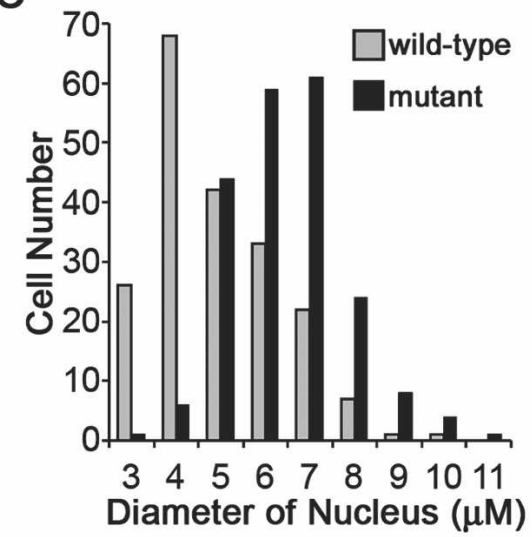

B

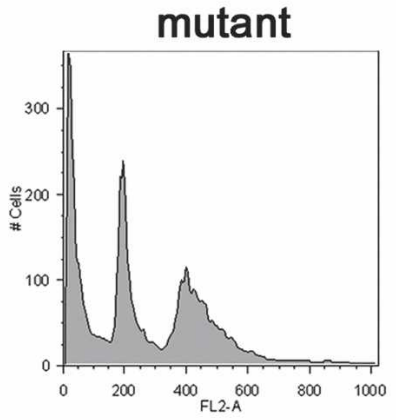

D

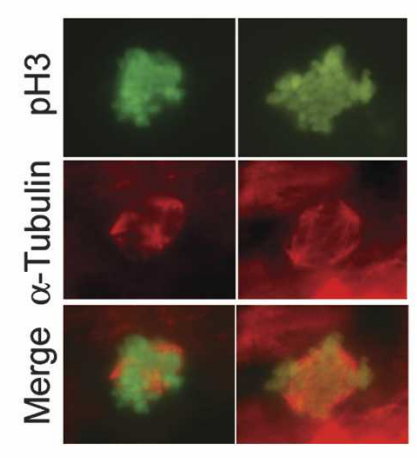

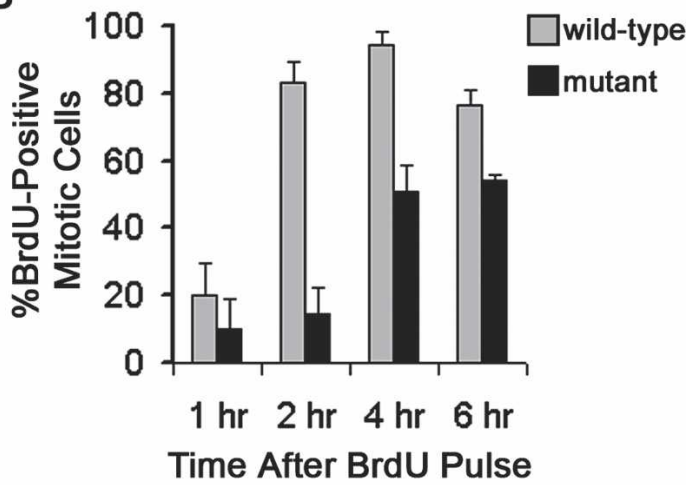

E

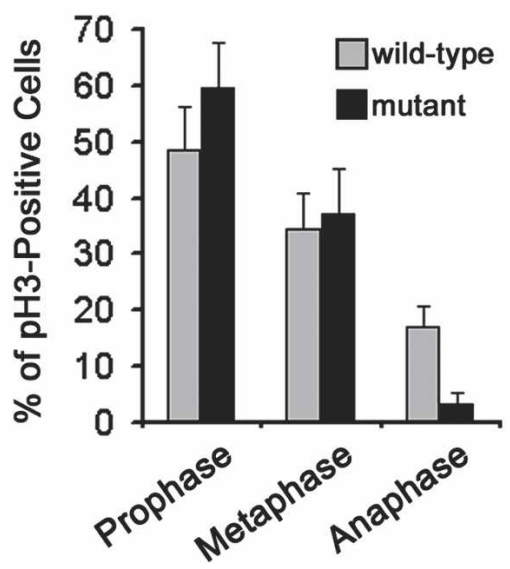

Figure 2. Dtl is required for normal cell cycle progression in zebrafish embryos. (A) Cell cycle profiles of cells from 32 -hpf hi3627 mutant $(-/-)$ and wild-type clutchmate $(+/+;+/-)$ zebrafish embryos showing an increase in $4 \mathrm{~N}$ and $>4 \mathrm{~N}$ cells in the mutants. $(B)$ The S-to-M transition was slower in hi3627 mutant (-/-) versus wild-type (+/+; +/-) clutchmates, as assessed by pulse labeling with BrdU, incubating for the indicated times, and determining the fraction of $\mathrm{pH} 3$-positive (mitotic) cells that had incorporated BrdU (mean $\pm \mathrm{SD}$; $n=5$ embryos). (C) Nuclear diameter was increased in hi3627 mutant (-/-) versus wild-type (+/+; $+/-) 32$-hpf clutchmates ( $n=40$ cells in each of five embryos). (D) Mitotic cells in 32-hpf hi3627 mutants stained with anti-pH3 (green) and anti- $\alpha$-tubulin (red) have supernumerary spindle poles. (E) Quantitation of prophase, metaphase, or anaphase cells (mean $\pm \mathrm{SD} ; n=3$ embryos), based on chromatin and spindle morphology, revealed a significant loss of anaphase cells in hi3627 mutants (-/-).

We have already shown that the unirradiated $d t 1$ mutants do not have an increased mitotic index, suggesting that the increase in $4 \mathrm{~N}$ cells represents a delay in $\mathrm{G} 2$ (Fig. 1F). To test this possibility, we pulsed 28-hpf embryos with BrdU; incubated the embryos 1, 2, 4, or $6 \mathrm{~h}$; and then counted the number of BrdU-positive mitotic cells in whole-mount stained embryos. The rate at which BrdU-positive cells entered mitosis reflects the duration of $S$ and G2. Between the first and second hours following the BrdU pulse, the percentage of $\mathrm{pH} 3$-positive cells with BrdU rose from $20 \%$ to $80 \%$ in wild-type embryos, indicating that the average duration of G2 in wildtype embryos is between 1 and $2 \mathrm{~h}$ (Fig. 2B). In contrast, very few of the BrdU-positive cells in the $d t l$ mutants had reached mitosis within $2 \mathrm{~h}$ of the pulse, and by $4 \mathrm{~h}$, less than half of the BrdU-positive cells had entered mitosis. Thus, Dtl loss causes a significant delay in the transition from $\mathrm{S}$ phase to mitosis. Taken together, these data demonstrate that there is a significant G2 delay in the $d t 1$ mutants. Importantly, a G2 delay is consistent with CDT1 deregulation, as CDT1 overexpression or geminin depletion is known to cause DNA damage, and the initiation of prolonged p53- or ATR/FANCA-dependent G2 checkpoints in human cells (Vaziri et al. 2003; Melixetian et al. 2004; Zhu and Dutta 2006).

We have also observed several other cellular abnormalities in the unirradiated $d t l$ mutants that are similar to phenotypes caused by deregulated CDT1 expression. First, we observed a significant increase in the diameter of nuclei in $d t l$ mutants compared with wild-type clutchmates (Fig. 2C). This has been observed in D. melanogaster, C. elegans, and human cells when geminin is depleted and in C. elegans embryos when CUL4 is knocked down (Zhong et al. 2003; Melixetian et al. 2004; Yanagi et al. 2005). Second, while the mitotic index of the $d t l$ mutants is not significantly different from wildtype controls (Fig. 1F), examination of anti-pH3/anti- $\alpha$ tubulin double-labeled cells in the mutant embryos revealed striking mitotic abnormalities. Specifically, mitotic cells within $d t l$ mutants frequently had multipolar 
spindles (Fig. 2D). This is in stark contrast to wild-type embryos in which, to date, we have not seen multipolar spindles (data not shown). Additionally, anaphase cells were significantly underrepresented in the $d t l$ mutants $(p<0.0005)$, as judged by quantifying the fraction of $\mathrm{pH} 3$ positive cells that were in prophase, metaphase, or anaphase based on chromatin and mitotic spindle morphology (Fig. 2E). These mitotic defects could be caused by the G2 delay/rereplication because prolonged G2 retention is known to allow centrosome overduplication, and the subsequent formation of multipolar spindles causes mitotic catastrophe and the loss of anaphase cells (Castedo et al. 2004; Dodson et al. 2004). Consistent with this notion, small interfering RNA (siRNA) knockdown of geminin in human cells causes centrosome overduplication in addition to rereplication and G2 checkpoint activation (Tachibana et al. 2005).

\section{DTL associates with the CUL4A-DDB1 E3 ubiquitin ligase}

The preceding analysis shows that unirradiated $d t 1 \mathrm{mu}-$ tant embryos display numerous defects including G2 delay, rereplication, enlarged nuclei, multipolar spindles, and a deficiency of anaphase cells. As we describe above, these cellular defects resemble those observed in human cells with the knockdown of geminin or the overexpression of their downstream target CDT1 (Vaziri et al. 2003; Melixetian et al. 2004; Tachibana et al. 2005; Zhu and Dutta 2006). Given these facts, we hypothesized that DTL is required for CDT1 inhibition during $S$ phase. This hypothesis was supported by the recent finding that Cdt2 (the S. pombe DTL homolog) is required for the Pcu4-Ddb1 E3 ubiquitin ligase (the $S$. pombe equivalent of CUL4-DDB1) to ubiquitinate an inhibitor of ribonucleotide reductase called Spd1 during S phase and after DNA damage (Liu et al. 2005). Therefore, we hypothesized that DTL is a component of the CUL4-DDB1 complex required for CDT1 ubiquitination.

To determine whether vertebrate DTL associates with the CUL4-DDB1 E3 ligase, we purified DTL complexes using the tandem affinity purification method (TAP) (Puig et al. 2001). We selected human cells for these studies because of the availability of antibodies against CUL4-DDB1 complex components. We generated a variant of HeLa-S3 cells that stably expressed an N-terminally tagged TAP-DTL fusion protein. This TAP-DTL was expressed at a comparable level to the endogenous DTL and showed the appropriate nuclear localization (Fig. 3A; Cheung et al. 2001; data not shown). We affinity-purified TAP-DTL, or the TAP control protein, and then screened for DDB1 and CUL4A by immunoblotting (Fig. 3A). In each case, DDB1 and CUL4A copurified with TAP-DTL, but not with TAP alone, indicating that DTL interacts with both of these proteins in human cells.

To verify that this complex exists in vivo, we conducted immunoprecipitation experiments with the endogenous DTL protein. For these experiments, we generated a mouse polyclonal antiserum against the C-ter-

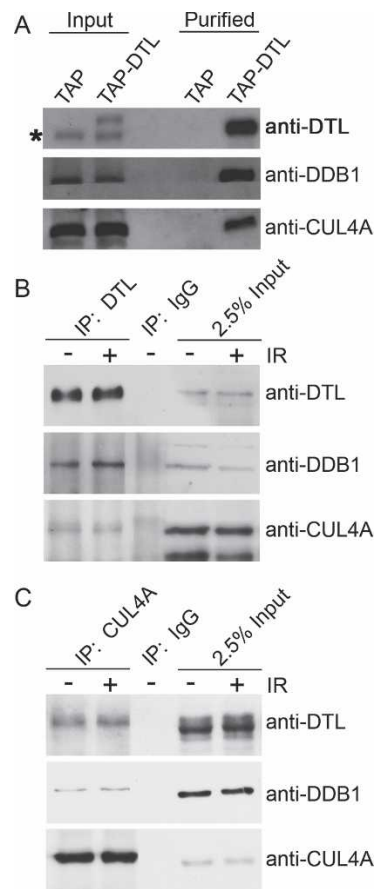

Figure 3. Human DTL interacts with the CUL4A-DDB1 complex. (A) TAP alone or TAP-DTL expressed in HeLa-S3 cells was purified from whole-cell lysates using a TAP procedure. Immunoblotting shows DTL, DDB1, and CUL4A in the input lysate copurified with TAP-DTL but not the TAP control. The asterisk indicates endogenous DTL. TEV protease cleavage caused a slight change in TAP-DTL's size. $(B, C)$ Whole-cell lysates were prepared from either unirradiated (-IR) or irradiated (+IR) HeLa cells, and endogenous DTL $(B)$ or CUL4A $(C)$ was immunoprecipitated. Input lysate and the immunoprecipitates were then screened for DTL, DDB1 and CUL4A proteins by immunoblotting.

minal half of the human DTL protein and confirmed that it detected an $\sim 80$ - $\mathrm{kDa}$ protein that was depleted in $\mathrm{HeLa}$ cells transfected with siRNA targeting the DTL mRNA (Fig. 4A). This anti-DTL polyclonal, but not control mouse, serum coimmunoprecipitated DTL, DDB1, and CUL4A from HeLa cells (Fig. 3B). We also performed reciprocal immunoprecipitation experiments with a polyclonal antibody against CUL4A, demonstrating that DTL and DDB1 both coimmunoprecipitate with CUL4A (Fig. 3C). We therefore conclude that DTL is a component of the CUL4A-DDB1 complex in human cells.

The CUL4-DDB1 complex ubiquitinates CDT1 upon DNA damage, but the mechanism by which the complex is activated is not completely understood. In S. pombe, IR exposure causes an increase in Cdt2 protein levels within $1 \mathrm{~h}$ (Liu et al. 2005); therefore, we tested whether DNA damage increased the abundance of DTL in human cells or stimulated the interaction of DTL with the CUL4-DDB1 complex. Exposing HeLa cells to IR $1 \mathrm{~h}$ before the immunoprecipitations did not cause an appreciable change in DTL protein levels overall or in the extent of interaction between DTL and CUL4DDB1 (Fig. 3B,C). Thus, unlike the Pcu4-Ddb1-Cdt2 
A
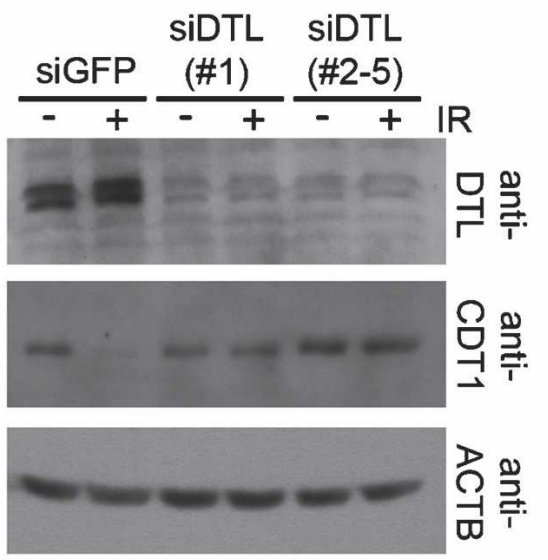

B

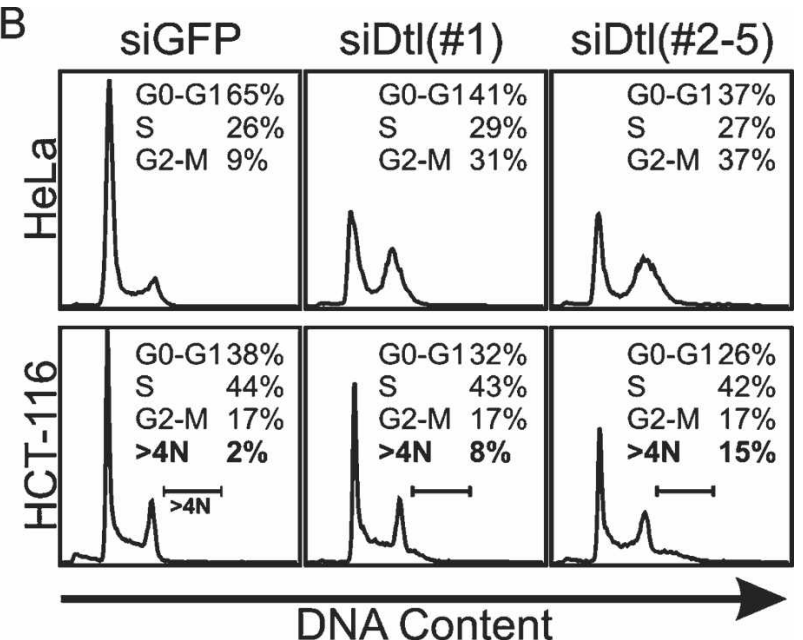

C

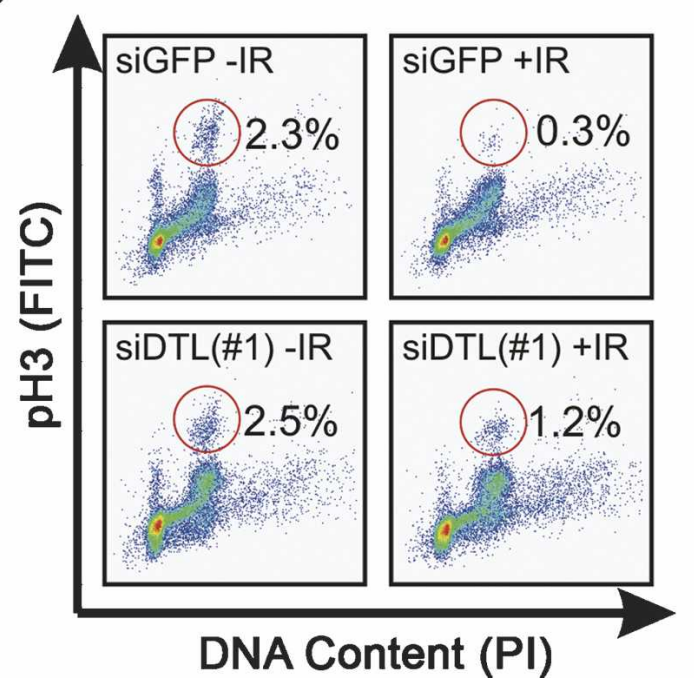

D

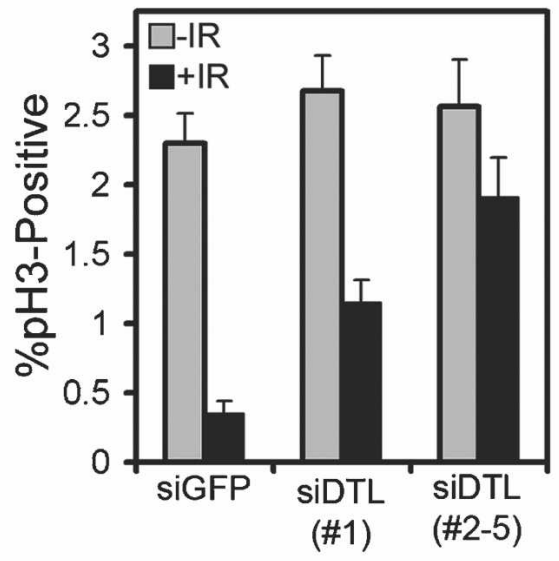

Figure 4. DTL is required for regulating CDT1 and activating the G2 checkpoint in human cells. $(A)$ HeLa cells were transfected with single siRNAs, siGFP, or siDTL(\#1), or a pool of four distinct siRNAs [siDTL(\#2-\#5)], and after $72 \mathrm{~h}$, the levels of DTL and CDT1, relative to the $\beta$-actin (ACTB) loading control, were determined by immunoblotting of whole-cell lysates. $(B)$ Cell cycle profiles of siDTL(\#1)- or siDTL(\#2-\#5)-transfected HeLa cells (72 h post-transfection) or HCT-116 cells (96 h post-transfection). (C,D) HeLa cells were transfected with siRNAs as described in $A$, and half of the cells were exposed to $10 \mathrm{~Gy}$ of ionizing radiation (+IR) and then incubated for $1 \mathrm{~h}$ before labeling with anti-pH3 and PI. $(C)$ The percentages of pH3-positive cells were calculating using FloJo software. $(D)$ Averages from four separate experiments are shown (mean $\pm \mathrm{SD} ; n=20,000$ counts in each experiment).

complex in S. pombe, the activation of CUL4-DDB1DTL does not involve an immediate change in DTL expression.

\section{DTL is required for CDT1 destruction in human cells}

Having shown that DTL binds to CUL4A-DDB1, we sought to determine whether DTL is required for this E3 ligase to target CDT1 for degradation. In the normal cell cycle, the CUL4A-DDB1 and SCF-SKP2 complexes both contribute to the ubiquitination and degradation of CDT1, and consequently the loss of CUL4-DDB1 alone is thought to be insufficient to affect CDT1 levels (Nishitani et al. 2006; Senga et al. 2006). In contrast, CDT1 degradation after DNA damage is solely depen- dent on the CUL4A-DDB1 ligase (Higa et al. 2003; Hu et al. 2004; Nishitani et al. 2006). Thus, to test whether DTL is required for the CUL4A-DDB1-dependent degradation of CDT1, we examined CDT1 levels in $\gamma$-irradiated HeLa cells in which the DTL protein was knocked down using siRNAs. A pool of four separate siRNAs [siDTL(\#2-\#5)] or a single, distinct siRNA [siDTL(\#1)] caused dramatic, but not complete, knockdown of DTL protein by $72 \mathrm{~h}$ after transfection in HeLa cells (Fig. 4A). Irradiation of HeLa cells that were transfected with a control siRNA (siGFP) caused a pronounced down-regulation of CDT1 protein within $1 \mathrm{~h}$. In contrast, CDT1 protein levels were completely unaffected by DNA damage in either the siDTL(\#1)- or siDTL(\#2-\#5)-transfected cells (Fig. 4A). These results demonstrate that DTL is 
required for the CUL4A-DDB1-dependent degradation of CDT1 after DNA damage.

\section{DTL is required for the normal cell cycle regulation} of CDT1 in human cells

The cell cycle defects that we have observed in zebrafish $d t 1$ mutants suggest that the Scf-Skp2 complex cannot completely compensate for the loss of Dtl in unperturbed cells. Thus, we questioned whether DTL is required for the normal cell cycle regulation of CDT1 in human cells. We found that siRNA knockdown of DTL in human cells yielded cell cycle defects that are consistent with a failure to appropriately down-regulate CDT1 and recapitulated the cell cycle defects we observed in the $d t l$ mutant zebrafish. Transfection of HeLa cells with the siDTL(\#2-\#5) pool caused a reproducible increase in both the level of CDT1 and in the percentage of cells in G2 (Fig. 4B). The G2 peak also showed a subtle $>4 \mathrm{~N}$ shoulder suggestive of partial rereplication. Transfection of the single siDTL(\#1) caused a less pronounced increase in $4 \mathrm{~N}$ cells and no detectable affect on CDT1 levels (Fig. 4A), suggesting that there is a subtle difference in the potency of siDTL(\#1) versus the siDTL(\#2-\#5) pool. In a second human cell line, HCT-116, these siRNAs displayed a similar difference in their relative efficacy but gave more pronounced phenotypes, yielding first an accumulation of G2 cells (72 h after the transfection) (data not shown) and subsequently the appearance of cells with $>4 \mathrm{~N}$ DNA content (96 h) (Fig. 4B). Thus, DTL is required for both CDT1 degradation and inhibition of rereplication during the normal cell cycle of human cells.

\section{DTL is required for the early G2/M checkpoint in human cells}

Having demonstrated that DTL is required to prevent rereplication in both zebrafish embryos and human cells, we then tested whether the G2/M checkpoint function of DTL was also conserved. These experiments were conducted using HeLa cells transfected with siGFP versus siDTL(\#1) or siDTL(\#2-\#5). For these experiments, the cells were treated with IR, and $1 \mathrm{~h}$ later, the fraction of pH3-positive cells was quantified by FACS (Fig. 4C,D). Without IR, the control- and siDTL-transfected cells had similar mitotic indexes (Fig. 4D). IR treatment caused an eightfold decrease in pH3-positive cells in the control siGFP-transfected cells (Fig. 4D), indicating that the early G2 checkpoint had been activated. In contrast, IR exposure caused only a 2.3 -fold or 1.3 -fold reduction in pH3-positive cells in siDTL(\#1)- or siDTL(\#2-\#5)-transfected cells, respectively (Fig. 4C,D). Thus, in a similar manner to the zebrafish, human DTL is required for the IR-induced, early G2 checkpoint response.

Cdt1 contributes to the cell cycle, but not the checkpoint, defects of the Dtl mutants

Loss of DTL causes CDT1 deregulation and G2 checkpoint failure, two seemingly unrelated phenotypes, in both zebrafish embryos and human cells. We wished to determine whether the inappropriate stabilization of Cdt 1 contributes to the early G2 checkpoint defect of $d t 1$ mutant zebrafish. To address this question, we used morpholino oligonucleotides that block the splicing of the zygotic pre-mRNAs to probe the interplay between $c d t 1$ and $d t 1$ in zebrafish. Consistent with Cdt1 being essential for DNA replication, injection of zebrafish embryos with 1 pmol of $c d t 1$ morpholino causes a highly penetrant arrest by $6 \mathrm{hpf}$ (60\% epiboly) (data not shown). This phenotype is much stronger than that observed in the $d t l$ morphants (Fig. 5A). Thus, to determine whether cdt 1 contributes to the $d t l$ mutant phenotypes, we titrated down the dose of injected cdt1 morpholino to cause partial knockdown. Injection of $200 \mathrm{fmol}$ of $c d t 1$ morpholino yielded embryos that lived to $28 \mathrm{hpf}$ but were severely apoptotic/necrotic and displayed a significant accumulation of cells in G1 (data not shown). A further reduction to $50 \mathrm{fmol}$ consistently yielded 28 -hpf embryos that had no significant cell cycle defect (Fig. 5A). Real-time PCR analysis shows that the level of mature cdt 1 mRNA was reduced by $60 \%$ in these morphants (Fig. 5C). As previously described, injection of the $d t 1$ morpholino causes a partial knockdown of the $d t 1$ mRNA (Fig. 5D) and accumulation of $4 \mathrm{~N}$ cells by $28 \mathrm{hpf}$ (Fig. 5A). When we injected $50 \mathrm{fmol}$ of $c d t 1$ morpholino together with the $d t 1$ morpholino, we observed near complete suppression of the $4 \mathrm{~N}$ cell accumulation in the $d t$ morphants (Fig. 5A). This strongly suggests that excessive Cdt1 causes the G2 defects arising in the $d t 1$ morphants.

In parallel with this cell cycle analysis, we also examined the checkpoint phenotypes of $c d t 1$ and $d t 1$ morphants (Fig. 5B). As we have described, the $d t l$ morphants had the same phenotype as the $d t t^{\text {hi3267 }}$ and $d t t^{\text {hi4 } 477}$ homozygous mutant embryos: pH3-positive cells were present at normal levels in unirradiated $d t$ morphants, but they were not down-regulated in response to IR treatment. Injection of $c d t 1$ morpholino alone had no effect on either the mitotic index of the unirradiated 28-hpf embryos or the checkpoint response after IR treatment. In the case of the $d t l / c d t 1$ double morphants, even though the reduced CDT1 dosage was sufficient to suppress the G2 progression defect of the $d t 1$ morphants (Fig. $5 \mathrm{~A})$, it had no effect on the defective IR-induced, G2 checkpoint response (Fig. 5B). This strongly suggests that the role of Dtl in the early G2 checkpoint is Cdt1 independent.

\section{Discussion}

In this study, we have used zebrafish as a model organism to study cell cycle control and DNA damage response. We first established that zebrafish embryos activate a robust G2/M DNA damage checkpoint within $1 \mathrm{~h}$ of IR treatment. Like the mammalian early G2/M checkpoint response, this G2 arrest is caffeine-sensitive, indicating that it is ATM/ATR-dependent. We conducted a genetic screen for zebrafish mutant embryos that failed to appropriately activate this checkpoint. From this 
A

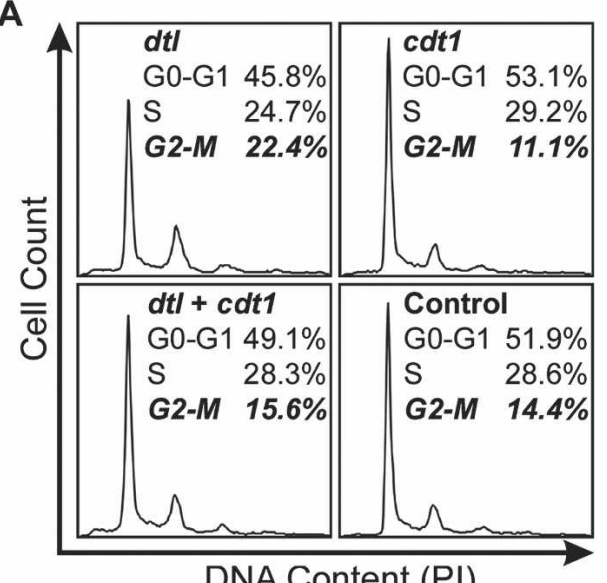

C

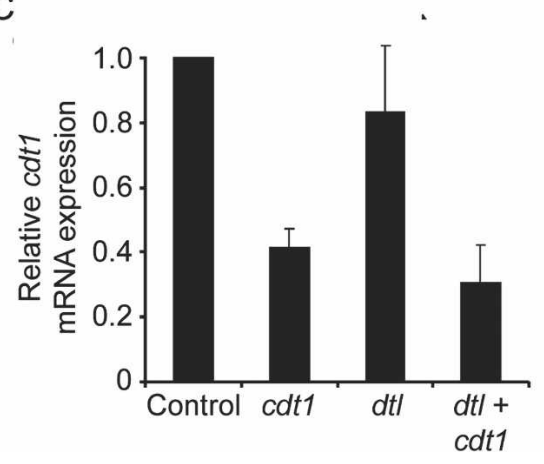

B
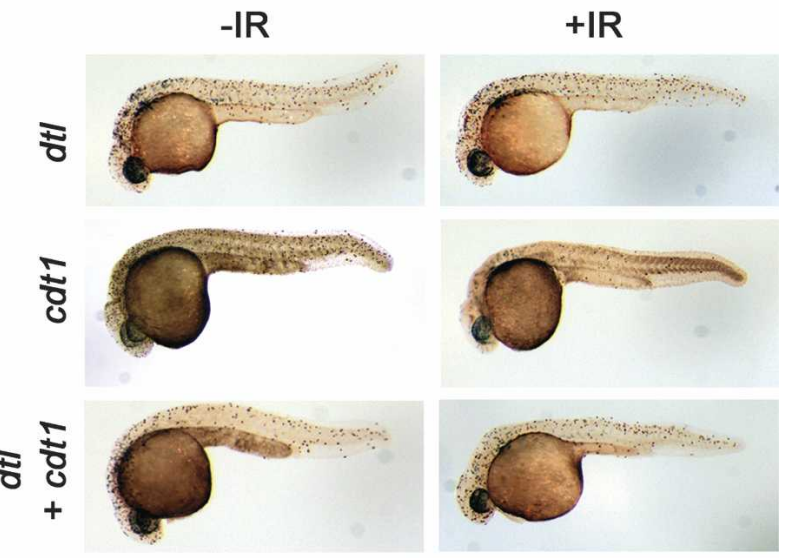

$\mathrm{D}$
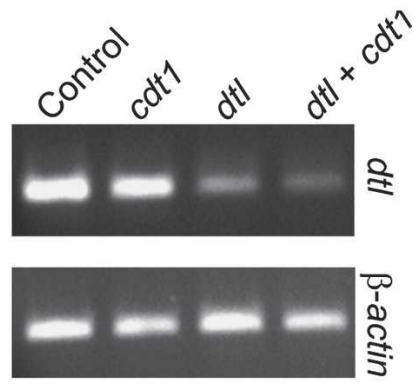

Figure 5. Knockdown of $c d t 1$ rescues $4 \mathrm{~N}$ accumulation in $d t 1$ morphants but not the $\mathrm{G} 2$ checkpoint defect. $(A)$ Zebrafish embryos were injected with vehicle (Control), $d t 1$ morpholino, $c d t 1$ morpholino, or both $(d t 1+c d t 1)$. Cell cycle profiles of cells dissociated from those morpholino-injected embryos at $28 \mathrm{hpf}$ are shown. (B) Mitotic cells in 28-hpf zebrafish embryos, which were morpholino-injected as in $A$, were labeled with an anti-pH3 antibody $1 \mathrm{~h}$ after the embryos were exposed to 15 Gy of IR. $(C, D)$ Total RNA was prepared from morpholino-injected embryos at $28 \mathrm{hpf}$ and, using $\beta$-actin as a control, were analyzed for $c d t 1$ mRNA by real-time PCR (C) $($ mean $\pm \mathrm{SD} ; n=4)$ or $d t 1$ mRNA by RT-PCR $(D)$.

screen, we identified two mutant alleles of the $d t l$ gene that cause G2/M checkpoint failure. Our subsequent analysis of both the zebrafish mutants and siRNA knockdown in human cells showed that DTL loss causes phenotypes that are conserved between zebrafish and humans. DTL is required for the rapid G2 checkpoint response and also normal cell cycle control, and these two functions appear to arise through the regulation of distinct downstream targets.

\section{A critical role for DTL in preventing rereplication through the regulation of CDT1}

The depletion of DTL causes rereplication, G2 delay, and the appearance of multipolar spindles. Strikingly, these phenotypes have all been observed in other systems in which CDT1 is inappropriately regulated. CDT1 inhibition by geminin and the UPS is critical for preventing de novo replication origin formation during $S$ phase; consequently, overexpressing CDT1 or depleting geminin causes rereplication (Vaziri et al. 2003; Melixetian et al. 2004; Zhu et al. 2004). Through unknown mechanisms, rereplication leads to DNA damage and the subsequent activation of prolonged G2/M checkpoints (Zhu and Dutta 2006). Furthermore, depletion of geminin causes centrosome overduplication that may also indirectly result from rereplication, since supernumerary centrosomes and multipolar spindles can result from extended G2 arrest (Dodson et al. 2004; Tachibana et al. 2005). Therefore, the array of phenotypes that we observed in undamaged DTL-depleted zebrafish embryos or human cells strongly suggested that DTL was an inhibitor of CDT1. Consistent with this notion, we found that knockdown of DTL in HeLa cells causes CDT1 overexpression and completely abrogates the DNA-damage-induced destruction of CDT1. We also demonstrated that DTL is part of the CUL4A-DDB1 E3 ubiquitin ligase that ubiquitinates CDT1 during $S$ phase and following DNA damage. Finally, knockdown of Cdt1 suppresses the cell cycle defects in Dtl-depleted zebrafish embryos. Together these data demonstrate that DTL is an essential component of the CUL4-DDB1 complex that targets CDT1 for destruction. Additionally, these data suggest that the cell cycle defects that we have observed in undamaged, DTL-depleted cells are entirely caused by the deregulation of CDT1. Contemporaneous with our 
study, others have shown that DTL/CDT2 is one of several DDB1- and CUL4-associated factors (DCAFs) and that DTL/CDT2 is required for CDT1 degradation (Angers et al. 2006; Higa et al. 2006; Jin et al. 2006).

\section{CUL4-DDB1-DTL is essential for preventing rereplication in vertebrates}

Our data provide insight into the essential mechanisms that prevent rereplication in human cells. Cul4 is necessary to prevent rereplication in C. elegans larvae, but recent reports have suggested that geminin or SCF-Skp2 compensates for the loss of CUL4-DDB1 to properly regulate CDT1 in vertebrates, suggesting that CUL4DDB1-dependent regulation of CDT1 during normal cell cycle progression is redundant (Zhong et al. 2003; Nishitani et al. 2006). We have demonstrated that loss of zebrafish Dtl causes overt rereplication, indicating that the Cul4-Ddb1-Dtl complex plays a nonredundant role in the regulation of $\mathrm{Cdt} 1$ in zebrafish embryos. Moreover, depletion of DTL in human HCT-116 and HeLa cells is also sufficient to cause rereplication. Thus, in addition to playing an essential role in DNA-damage-induced CDT1 destruction, the CUL4-DDB1-DTL complex plays an indispensable role in the regulation of CDT1 during normal cell cycle progression in vertebrates.

Geminin depletion or CDT1 overexpression causes the activation of robust, prolonged G2 checkpoints and dramatic decreases in the numbers of pH3-positive cells (Vaziri et al. 2003; Zhu and Dutta 2006). In contrast, we find that DTL down-regulation can cause CDT1 stabilization and G2 defects, but the cells are still able to enter mitosis. This disparity could result from the presence of low levels of residual DTL in our knockdown cells or differences in the degree of rereplication or DNA damage response in the various cell types used for these experiments. Alternatively, Dtl-deficient cells may continue entering mitosis because they have a reduced ability to initiate or maintain the prolonged G2/M DNA damage checkpoint. This is an intriguing possibility given that $d t l$ mutants also fail to halt mitotic entry following exposure to IR.

\section{DTL is required for the early G2/M DNA damage checkpoint}

We originally identified $d t l$ as a gene required in zebrafish embryos for the initiation of an IR-induced G2/M checkpoint; subsequently, we also demonstrated that depletion of DTL abrogates the early G2/M checkpoint in human cells. However, DTL loss also triggers a G2 delay that is consistent with the activation of a prolonged G2/M checkpoint. Thus, our data show that DTL depletion in zebrafish or human cells both abrogates and activates G2/M checkpoints. This is seemingly contradictory, but there is good reason to believe that this reflects the influence of DTL loss on two distinct G2 checkpoint pathways: an early checkpoint and a prolonged checkpoint. As we described above, rereplicationinduced DNA damage is known to promote G2 arrest through activation of the prolonged p53- or ATR/FANCdependent checkpoints (Vaziri et al. 2003; Zhu and Dutta 2006). In contrast, the rapid IR-induced G2 arrest for which DTL is required has been shown to be p53- and ATR/FANC-independent. Therefore, we propose that DTL is required for an early G2/M checkpoint that is distinct from the prolonged G2/M checkpoints initiated by rereplication.

While Cdt1 deregulation profoundly affects the cell cycle, there is no evidence that it prevents the activation of the early G2/M checkpoint. However, it is possible that the robust, prolonged G2/M checkpoints activated by geminin depletion or CDT1 overexpression preclude detection of an early G2/M checkpoint defect. Therefore, as a first step toward understanding the role of Dtl in the early G2/M checkpoint, we used the zebrafish to assess whether the checkpoint failure is a consequence of Cdt 1 deregulation. In these experiments, a reduction in Cdt 1 dosage was sufficient to rescue the cell cycle defect of $d t 1$ morphant zebrafish, but it had no appreciable affect on the G2/M checkpoint failure. This strongly suggests that the checkpoint defect reflects a Cdt1-independent role of Dtl. One caveat of this experiment is that it necessarily involves partial Cdt1 knockdown, since Cdt1 loss is lethal at $65 \%$ epiboly. Thus, it is formally possible that the residual Cdt1 in these morphants is sufficient to inhibit the G2 checkpoint. As a reciprocal approach, we attempted to assess the G2 checkpoint in zebrafish embryos in which Cdt1 is overexpressed (C.L. Sansam and J.L. Shepard, unpubl.). However, injection of $c d t 1$ mRNA caused lethality at $65 \%$ epiboly, underscoring the importance of maintaining appropriate Cdt1 levels (too much or too little is equally lethal) but precluding analysis of the G2 checkpoint. Since a reduction in zebrafish Cdt1 rescued the cell cycle, but not the checkpoint defect of $d t 1$ morphants, and there was no a priori evidence that CDT1 deregulation promotes DNA damage checkpoint failure, we hypothesize that the G2 checkpoint defect represents a CDT1-independent function of DTL (Fig. 6).

At a broader level, the essential function of DTL in the early G2/M checkpoint could reflect its role in the CUL4-DDB1 complex (presumably targeting one or more other substrates) or it could be CUL4-DDB1 independent. We favor the former model because there is strong evidence that the CUL4-DDB1 ligase plays multiple roles in the DNA damage response. We, and others, have shown that the DNA-damage-induced degradation of CDT1 or Spd1 is absolutely dependent on the human (CUL4-DDB1-DTL) or S. pombe (Pcu4-Ddb1-Cdt2) versions of the DTL-containing complex (Liu et al. 2005; Higa et al. 2006; Jin et al. 2006). Additionally, CUL4DDB1 ligases that include two other substrate recognition proteins, CSA or DDB2, are also modulated by DNA damage and play essential roles in the repair of ultraviolet (UV)-damaged DNA. Given these observations, we attempted to test whether the CUL4-DDB1 core complex is required for the early G2 checkpoint by knocking down DDB1 in zebrafish and human cells. However, the resulting phenotypes (lethality at $60 \%$ epiboly or complete G2 arrest and loss of pH3-positive cells) precluded 


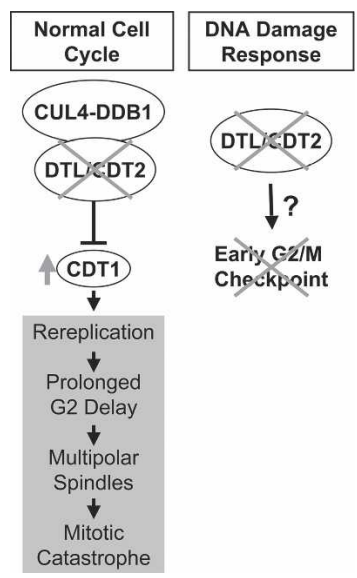

Figure 6. DTL is required both for the down-regulation of CDT1 during S phase and for the activation of the early G2/M checkpoint. The CUL4-DDB1-DTL complex inhibits CDT1 during S phase. Loss of DTL causes inappropriate expression of CDT1 during $S$ phase, leading to rereplication, prolonged G2 checkpoint activation, multipolar spindles, and mitotic catastrophe. DTL loss also prevents the activation of the IR-induced early G2/M checkpoint in a CDT1-independent manner.

examination of the G2 checkpoint (C.L. Sansam and J.L. Shepard, unpubl.). Notably, these defects were much stronger than those resulting from DTL knockdown. This could reflect differences in the efficiency of knockdown, or that DDB1 is required for more functions than DTL. The latter possibility seems likely since DDB1 is a core component of the CUL4 E3 ligase and DTL is just one of multiple specificity factors for this complex (Angers et al. 2006; Jin et al. 2006). Clearly, additional studies will be required to understand how DTL contributes to the rapid G2 checkpoint, and if it indeed reflects a novel role for the CUL4-DDB1-DTL complex, identify the relevant target(s).

\section{Materials and methods}

G2 checkpoint assays

To initiate a DNA damage response, zebrafish embryos and HeLa cells were exposed to 15 Gy or 10 Gy of IR, respectively, from a ${ }^{60} \mathrm{Co}$ source. One hour after irradiation, the zebrafish embryos or HeLa cells were prepared for $\mathrm{pH} 3$ analysis. The procedure for whole-mount immunostaining of zebrafish embryos with anti-pH3 (Santa Cruz Biotechnology; sc8656R) was previously described (Shepard et al. 2005). For FACS analysis, embryos were sorted based on developmental phenotypes into mutant (-/-) or wild-type $(+/+;+/-)$ groups, and pools of 20 embryos were triturated in $0.25 \%$ Trypsin/ $1 \mathrm{mM}$ EDTA. Suspensions of zebrafish embryo cells or HeLa cells were prepared for $\mathrm{pH} 3 /$ propidium iodide FACS analysis according to a previously described protocol (Xu et al. 2002). To test whether caffeine could inhibit the zebrafish G2 checkpoint, 32-hpf embryos were placed in $2 \mathrm{mM}$ caffeine and incubated for $30 \mathrm{~min}$ at $28.5^{\circ} \mathrm{C}$ before the IR/pH3 assay.

Zebrafish maintenance, collection, and genotyping

Zebrafish were maintained as described (Amsterdam et al. 2004). For the screen, heterozygous insertion carriers were in- crossed, and 24-hpf embryos were dechorionated and incubated in 1-phenyl-2-thiourea (PTU, $0.003 \%$ ) to inhibit pigmentation. At 32 hpf, 60 embryos from each clutch were subjected to G2/M checkpoint analysis. The $d t \mathrm{t}^{\text {hi3627 }}$ embryos that retained $\mathrm{pH} 3$ staining post-radiation were individually PCR-genotyped (hi3627 forward [F], CCCTTCTTCGTTGGCTACAG; hi3627 reverse [R], GTCTCTGCTGGACGGCTATG; provirus reverse, TGATCTCGAGTTCCTTGGGAGGGTCTCCTC).

\section{Morpholino injections}

Splice-blocking morpholinos (Gene Tools) were injected to reduce expression of $d t$ l-exon 4 (AAAGTACCTTTTTTACTG CATCTTG) or $c d t 1$-exon 3 (TGAGCAGCTATCCTCACC GTTCCTG). Knockdown of the $d t 1$ mRNA was confirmed by RT-PCR (F, AGCACTGGAGGCAGAGATGG; and R, GATAG GCCTGTAGAGGCAGG), and knockdown of the $c d t 1$ mRNA was measured by two-step real-time RT-PCR with SYBR Green I Dye (Applied Biosystems) (F, CTGAGACACAGCCTGCA ACT; and R, GGGCACATCCTGAGCTAAA) and normalized against zebrafish $\beta$-actin (F, CATCAGCATGGCTTCTGCTC TGTATGG; and R, GACTTGTCAGTGTACAGAGACACC CTG).

\section{Cell cycle analysis}

For DNA content analysis, zebrafish cell suspensions were prepared as described above for the pH3/PI FACS procedure. Suspensions of zebrafish embryo cells, HeLa cells, or HCT-116 cells were processed for DNA content FACS analysis as described (Pozarowski and Darzynkiewicz 2004). The cells were analyzed using a FACScan machine (Becton-Dickinson), and the data were analyzed using ModFit LT software (Verity Software). The BrdU pulse experiments and whole-mount staining of embryos with anti-pH3 and anti- $\alpha$-tubulin were performed as previously described (Shepard et al. 2005).

\section{Cell culture and siRNA transfections}

HeLa cells were maintained in DMEM with $10 \%$ fetal bovine serum. HeLa-S3 cells were maintained in SMEM with 10\% bovine calf serum. The following siRNAs were generated by Dharmacon: siGFP, UCCCGGCUAUGUGCAGGAGUU; siDTL(\#1), AAGGUUCCUGGUGAACUUAAAUU; siDTL(\#2), ACUCCU ACGUUCUCUAUUAUU; siDTL(\#3), GUAUGGGAUUUACG UAAGAUU; siDTL(\#4), AGAAGGCUUUGUUCGAUUGUU; siDTL(\#5), GCUAAUUGCACAGACGAUAUU; and siDDB1, GGACCUGCUGUUUAUCUUGUU. The individual siRNAs were transfected at $100 \mathrm{nM}$, while each siRNA in the siDTL/\#2\#5) pool was at $25 \mathrm{nM}$. All siRNAs were transfected with Oligofectamine (Invitrogen) according to the manufacturer's instructions.

\section{Generation of anti-DTL antiserum}

To generate polyclonal antiserum for immunoblotting and immunoprecipitation of human DTL, a $6 \times$ His-tagged fragment of human DTL (NP_057532, amino acids 388-690) was expressed in bacteria, purified over $\mathrm{Ni}^{2+}$-nitrilotriacetic acid-agarose resin (Qiagen), and used to immunize BALB/c mice. The resultant polyclonal antiserum was tested for its ability to detect endogenous Dtl protein in HeLa cells, and the specificity of the antiserum was confirmed using lysates from DTL siRNA-transfected HeLa cells. 
Protein preparation, TAP-tag purification, and immunoprecipitation

To generate the TAP-DTL expression vector, the human DTL cDNA was PCR-amplified and cloned into pENTR/D-TOPO (Invitrogen) according to the manufacturer's instructions. The cDNA was then transferred to the pDS_MSCVmacs4-TAP-XB retroviral expression vector (ATCC \#10326361) using the standard Invitrogen Gateway LR reaction. TAP or TAP-DTL retrovirus, which was packaged using Phoenix cells as described (Fassati et al. 1994), was used to infect HeLa-S3 cells, and lowexpressing cells were selected by FACS sorting cells surfacelabeled with FITC anti-CD4 (Miltenyi Biotec). Expression of TAP or TAP-DTL was confirmed using peroxidase anti-peroxidase (Sigma) or DTL antiserum. Whole-cell lysates were prepared with $0.1 \%$ NP-40 lysis buffer and subjected to the previously described TAP-tag purification procedure (Puig et al. 2001). Coimmunoprecipitations were performed using $0.1 \%$ NP-40 lysis buffer and antibodies against CUL4A (Rockland, rabbit) or DTL. Immunoblotting was performed using antiDDB1 (Genetex; rabbit), anti-CUL4A, or anti-DTL.

\section{Acknowledgments}

We thank Mike Yaffe and members of the Hopkins and Lees laboratories for helpful discussions during this study and the preparation of this manuscript. This work was supported by a grant from the NIH (RO1-RR12589) to N.H. and fellowships to C.L.S. (Ruth L. Kirschstein NRSA), A.I. (American Italian Cancer Foundation), J.L.S., and K.L. (Anna Fuller Fellowships). J.A.L. is a Daniel K. Ludwig Scholar.

\section{References}

Amsterdam, A., Nissen, R., Sun, Z., Swindell, E., Farrington, S. and Hopkins, N. 2004. Identification of 315 genes essential for early zebrafish development. Proc. Natl. Acad. Sci. 101: $12792-12797$.

Angers, S., Li, T., Yi, X., MacCoss, M.J., Moon, R.T., and Zheng, N. 2006. Molecular architecture and assembly of the DDB1CUL4A ubiquitin ligase machinery. Nature doi:10.1038/nature05175.

Arias, E. and Walter, J. 2005. PCNA functions as a molecular platform to trigger Cdt1 destruction and prevent re-replication. Nat. Cell Biol. 8: 84-90.

Bell, S. and Dutta, A. 2002. DNA replication in eukaryotic cells. Annu. Rev. Biochem. 71: 333-374.

Brown, E. and Baltimore, D. 2003. Essential and dispensable roles of ATR in cell cycle arrest and genome maintenance. Genes \& Dev. 17: 615-628.

Castedo, M., Perfettini, J., Roumier, T., Andreau, K., Medema, R., and Kroemer, G. 2004. Cell death by mitotic catastrophe: A molecular definition. Oncogene 23: 2825-2837.

Cheung, W., Chu, A., Chu, P., and Ip, N. 2001. Cloning and expression of a novel nuclear matrix-associated protein that is regulated during the retinoic acid-induced neuronal differentiation. J. Biol. Chem. 276: 17083-17091.

Dodson, H., Bourke, E., Jeffers, L., Vagnarelli, P., Sonoda, E., Takeda, S., Earnshaw, W., Merdes, A., and Morrison, C. 2004. Centrosome amplification induced by DNA damage occurs during a prolonged G2 phase and involves ATM. EMBO I. 23: 3864-3873.

Fassati, A., Takahara, Y., Walsh, F.S., and Dickson, G. 1994 Production of high titre helper-free recombinant retroviral vectors by lipofection. Nucleic Acids Res. 22: 1117-1118.
Freie, B., Ciccone, S., Li, X., Plett, P., Orschell, C., Srour, E., Hanenberg, H., Schindler, D., Lee, S., and Clapp, D. 2004. A role for the Fanconi anemia $\mathrm{C}$ protein in maintaining the DNA damage-induced G2 checkpoint. J. Biol. Chem. 279: 50986-50993.

Golling, G., Amsterdam, A., Sun, Z., Antonelli, M., Maldonado, E., Chen, W., Burgess, S., Haldi, M., Artzt, K., Farrington, S., et al. 2002. Insertional mutagenesis in zebrafish rapidly identifies genes essential for early vertebrate development. Nat. Genet. 31: 135-140.

Groisman, R., Polanowska, J., Kuraoka, I., Sawada, J., Saijo, M., Drapkin, R., Kisselev, A., Tanaka, K., and Nakatani, Y. 2003. The ubiquitin ligase activity in the DDB2 and CSA complexes is differentially regulated by the COP9 signalosome in response to DNA damage. Cell 113: 357-367.

Hendzel, M., Wei, Y., Mancini, M., Van Hooser, A., Ranalli, T., Brinkley, B., Bazett-Jones, D., and Allis, C. 1997. Mitosisspecific phosphorylation of histone $\mathrm{H} 3$ initiates primarily within pericentromeric heterochromatin during G2 and spreads in an ordered fashion coincident with mitotic chromosome condensation. Chromosoma 106: 348-360.

Higa, L., Mihaylov, I., Banks, D., Zheng, J., and Zhang, H. 2003. Radiation-mediated proteolysis of CDT1 by CUL4-ROC1 and CSN complexes constitutes a new checkpoint. Nat. Cell Biol. 5: 1008-1015.

Higa, L.A., Banks, D., Wu, M., Kobayashi, R., Sun, H., and Zhang, H. 2006. L2DTL/CDT2 interacts with the CUL4/ DDB1 complex and PCNA and regulates CDT1 proteolysis in response to DNA damage. Cell Cycle 5: 1675-1680.

Hu, J., McCall, C., Ohta, T., and Xiong, Y. 2004. Targeted ubiquitination of CDT1 by the DDB1-CUL4A-ROC1 ligase in response to DNA damage. Nat. Cell Biol. 6: 1003-1009.

Jin, J., Arias, E.E., Chen, J., Harper, J.W., and Walter, J.C. 2006. A family of diverse Cul4-Ddb1-interacting proteins includes $\mathrm{Cdt} 2$, which is required for $\mathrm{S}$ phase destruction of the replication factor Cdt1. Mol. Cell 23: 709-721.

Kastan, M. and Bartek, J. 2004. Cell-cycle checkpoints and cancer. Nature 432: 316-323.

Kurzik-Dumke, U., Neubauer, M., and Debes, A. 1996. Identification of a novel Drosophila melanogaster heat-shock gene, lethal(2)denticleless [1(2)dtl], coding for an 83-kDa protein. Gene 171: 163-170.

Lee, J., Kumagai, A., and Dunphy, W. 2001. Positive regulation of Weel by Chk1 and 14-3-3 proteins. Mol. Biol. Cell 12: $551-563$

Li, A. and Blow, J. 2004. Cdt1 downregulation by proteolysis and geminin inhibition prevents DNA re-replication in Xenopus. EMBO J. 24: 395-404.

Liu, Q., Guntuku, S., Cui, X., Matsuoka, S., Cortez, D., Tamai, K., Luo, G., Carattini-Rivera, S., DeMayo, F., Bradley, A., et al. 2000. Chk1 is an essential kinase that is regulated by Atr and required for the G(2)/M DNA damage checkpoint. Genes \& Dev. 14: 1448-1459

Liu, E., Li, X., Yan, F., Zhao, Q., and Wu, X. 2004. Cyclindependent kinases phosphorylate human Cdt1 and induce its degradation. J. Biol. Chem. 279: 17283-17288.

Liu, C., Poitelea, M., Watson, A., Yoshida, S.-H., Shimoda, C., Holmberg, C., Nielsen, O., and Carr, A. 2005. Transactivation of Schizosaccharomyces pombe cdt2 ${ }^{+}$stimulates a Pcu4-Ddb1-CSN ubiquitin ligase. EMBO J. 24: 3940-3951.

McGarry, T. and Kirschner, M. 1998. Geminin, an inhibitor of DNA replication, is degraded during mitosis. Cell 93: 10431053.

Melixetian, M., Ballabeni, A., Masiero, L., Gasparini, P., Zamponi, R., Bartek, J., Lukas, J., and Helin, K. 2004. Loss of Geminin induces rereplication in the presence of functional 
p53. J. Cell Biol. 165: 473-482.

Nishitani, H., Taraviras, S., Lygerou, Z., and Nishimoto, T. 2001. The human licensing factor for DNA replication Cdt1 accumulates in G1 and is destabilized after initiation of Sphase. J. Biol. Chem. 276: 44905-44911.

Nishitani, H., Sugimoto, N., Roukos, V., Nakanishi, Y., Saijo, M., Obuse, C., Tsurimoto, T., Nakayama, K., Nakayama, K., Fujita, M., et al. 2006. Two E3 ubiquitin ligases, SCF-Skp2 and DDB1-Cul4, target human Cdt1 for proteolysis. EMBO J. 25: 1126-1136.

Peng, C., Graves, P., Thoma, R., Wu, Z., Shaw, A., and PiwnicaWorms, H. 1997. Mitotic and G2 checkpoint control: Regulation of 14-3-3 protein binding by phosphorylation of Cdc25C on serine-216. Science 277: 1501-1505.

Pozarowski, P. and Darzynkiewicz, Z. 2004. Analysis of cell cycle by flow cytometry. Methods Mol. Biol. 281: 301-311.

Puig, O., Caspary, F., Rigaut, G., Rutz, B., Bouveret, E., BragadoNilsson, E., Wilm, M., and Seraphin, B. 2001. The tandem affinity purification (TAP) method: A general procedure of protein complex purification. Methods 24: 218-229.

Raleigh, J. and O'Connell, M. 2000. The G(2) DNA damage checkpoint targets both Weel and Cdc25. J. Cell Sci. 113: 1727-1736.

Sanchez, Y., Wong, C., Thoma, R., Richman, R., Wu, Z., Piwnica-Worms, H., and Elledge, S. 1997. Conservation of the Chk1 checkpoint pathway in mammals: Linkage of DNA damage to Cdk regulation through Cdc25. Science 277: 1497-1501.

Sarkaria, J., Busby, E., Tibbetts, R., Roos, P., Taya, Y., Karnitz, L., and Abraham, R. 1999. Inhibition of ATM and ATR kinase activities by the radiosensitizing agent, caffeine. Cancer Res. 59: 4375-4382.

Senga, T., Sivaprasad, U., Zhu, W., Park, J., Arias, E., Walter, J., and Dutta, A. 2006. PCNA is a cofactor for Cdt 1 degradation by CUL4/DDB1-mediated N-terminal ubiquitination. $I$. Biol. Chem. 281: 6246-6252.

Shepard, J., Amatruda, J., Stern, H., Subramanian, A., Finkelstein, D., Ziai, J., Finley, K., Pfaff, K., Hersey, C., Zhou, Y., et al. 2005. A zebrafish bmyb mutation causes genome instability and increased cancer susceptibility. Proc. Natl. Acad. Sci. 102: 13194-13199.

Skowyra, D., Craig, K., Tyers, M., Elledge, S., and Harper, J. 1997. F-box proteins are receptors that recruit phosphorylated substrates to the SCF ubiquitin-ligase complex. Cell 91: 209-219.

Smits, V., Klompmaker, R., Arnaud, L., Rijksen, G., Nigg, E., and Medema, R. 2000. Polo-like kinase-1 is a target of the DNA damage checkpoint. Nat. Cell Biol. 2: 672-676.

Tachibana, K.-E., Gonzalez, M., Guarguaglini, G., Nigg, E., and Laskey, R. 2005. Depletion of licensing inhibitor geminin causes centrosome overduplication and mitotic defects. EMBO Rep. 6: 1052-1057.

Takai, H., Tominaga, K., Motoyama, N., Minamishima, Y., Nagahama, H., Tsukiyama, T., Ikeda, K., Nakayama, K., Nakanishi, M., and Nakayama, K. 2000. Aberrant cell cycle checkpoint function and early embryonic death in $\mathrm{Chk} 1^{-/-}$ mice. Genes \& Dev. 14: 1439-1447.

Taylor, W. and Stark, G. 2001. Regulation of the G2/M transition by p53. Oncogene 20: 1803-1815.

Vaziri, C., Saxena, S., Jeon, Y., Lee, C., Murata, K., Machida, Y., Wagle, N., Hwang, D., and Dutta, A. 2003. A p53-dependent checkpoint pathway prevents rereplication. Mol. Cell 11: 997-1008.

Wertz, I., O'Rourke, K., Zhang, Z., Dornan, D., Arnott, D., Deshaies, R., and Dixit, V. 2004. Human De-etiolated-1 regulates c-Jun by assembling a CUL4A ubiquitin ligase. Science
303: $1371-1374$.

Wohlschlegel, J., Dwyer, B., Dhar, S., Cvetic, C., Walter, J., and Dutta, A. 2000. Inhibition of eukaryotic DNA replication by geminin binding to Cdt1. Science 290: 2309-2312.

Xu, B., Kim, S., Lim, D., and Kastan, M. 2002. Two molecularly distinct $G(2) / M$ checkpoints are induced by ionizing irradiation. Mol. Cell. Biol. 22: 1049-1059.

Yanagi, K., Mizuno, T., Tsuyama, T., Tada, S., Iida, Y., Sugimoto, A., Eki, T., Enomoto, T., and Hanaoka, F. 2005. Caenorhabditis elegans geminin homologue participates in cell cycle regulation and germ line development. J. Biol. Chem. 280: 19689-19694.

$\mathrm{Yu}, \mathrm{X}$. and Chen, J. 2004. DNA damage-induced cell cycle checkpoint control requires CtIP, a phosphorylation-dependent binding partner of BRCA1 C-terminal domains. Mol. Cell. Biol. 24: 9478-9486.

Zhao, H., Watkins, J., and Piwnica-Worms, H. 2002. Disruption of the checkpoint kinase 1 /cell division cycle $25 \mathrm{~A}$ pathway abrogates ionizing radiation-induced S and G2 checkpoints. Proc. Natl. Acad. Sci. 99: 14795-14800.

Zhong, W., Feng, H., Santiago, F., and Kipreos, E. 2003. CUL-4 ubiquitin ligase maintains genome stability by restraining DNA-replication licensing. Nature 423: 885-889.

Zhu, W. and Dutta, A. 2006. An ATR- and BRCA1-mediated Fanconi Anemia pathway is required for activating the G2/M checkpoint and DNA damage repair upon rereplication. Mol. Cell. Biol. 26: 4601-4611.

Zhu, W., Chen, Y., and Dutta, A. 2004. Rereplication by depletion of geminin is seen regardless of p53 status and activates a G2/M checkpoint. Mol. Cell. Biol. 24: 7140-7150. 


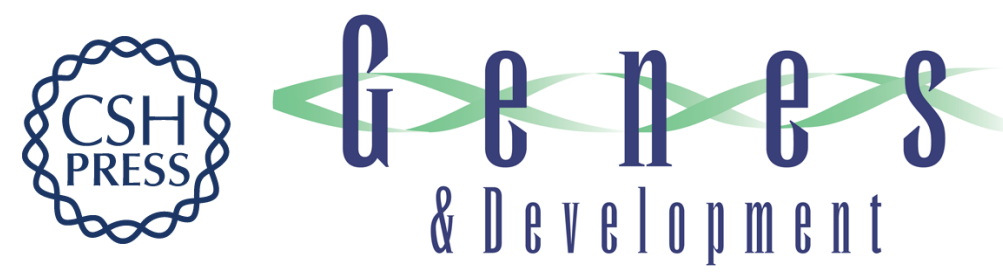

\section{DTL/CDT2 is essential for both CDT1 regulation and the early G2/M checkpoint}

Christopher L. Sansam, Jennifer L. Shepard, Kevin Lai, et al.

Genes Dev. 2006, 20: originally published online November 3, 2006

Access the most recent version at doi:10.1101/gad.1482106

References

This article cites 50 articles, 26 of which can be accessed free at:

http://genesdev.cshlp.org/content/20/22/3117.full.html\#ref-list-1

\section{License}

Email Alerting

Receive free email alerts when new articles cite this article - sign up in the box at the top Service right corner of the article or click here.

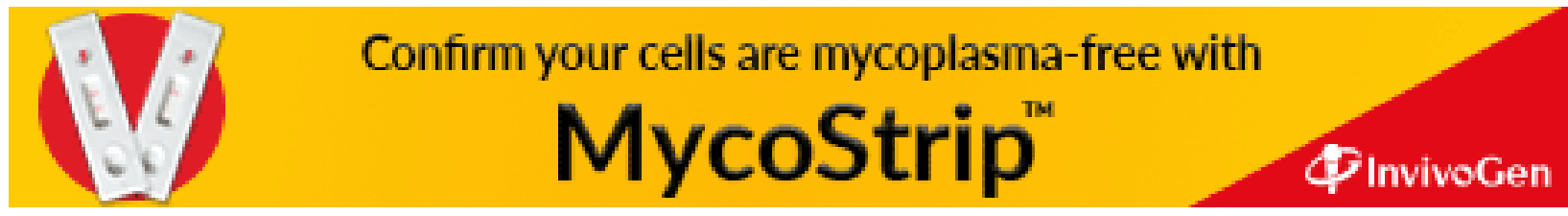

